\title{
Effects of onabotulinumtoxinA on cardiac function following intradetrusor injections
}

\author{
Ulrich Mehnert ${ }^{1}$, Laetitia M. de Kort ${ }^{2}$, Jens Wöllner ${ }^{1,3}$, Marko Kozomara ${ }^{1,4}$, \\ Gommert A. van Koeveringe ${ }^{5}$, Thomas M. Kessler ${ }^{1}$
}

\author{
1 Neuro-Urology, Spinal Cord Injury Center \& Research, Balgrist University Hospital, University of \\ Zürich, Zürich, Switzerland \\ 2 Department of Urology, Utrecht University Medical Center, Utrecht, The Netherlands \\ 3 Neuro-Urology, Swiss Paraplegic Center, Nottwil, Switzerland \\ 4 Department of Urology, University Hospital Zürich, Zürich, Switzerland \\ 5 Department of Urology, Maastricht University Medical Center, Maastricht, The Netherlands

$\begin{array}{ll}\text { Corresponding author: } & \text { Ulrich Mehnert, MD, FEBU } \\ & \text { Neuro-Urology } \\ & \text { Spinal Cord Injury Center \& Research } \\ & \text { University of Zürich } \\ & \text { Balgrist University Hospital } \\ & \text { Forchstrasse } 340 \\ & \text { 8008 Zürich } \\ & \text { Switzerland } \\ & \text { Tel.: +41 } 443865668 \\ \text { Fax: }+41443863909 \\ \text { ulrich.mehnert@paralab.balgrist.ch }\end{array}$

(C) 2016. This manuscript version is made available under the Elsevier user license http://www.elsevier.com/open-access/userlicense/1.0/ 


\section{ABSTRACT}

OnabotulinumtoxinA intradetrusor injections are considered a highly effective localized therapy for refractory detrusor overactivity. However, despite evidence for distant systemic effects of onabotulinumtoxinA, little is known on potential systemic side effects following intradetrusor injections. Given that onabotulinumtoxinA is a highly potent toxin this is an important safety issue specifically with regard to repeat injections and parallel treatments with botulinum toxin. Hence, it was the purpose of this prospective study to investigate, using heart rate variability (HRV) analysis, whether onabotulinumtoxinA causes systemic effects on cardiac function following intradetrusor injections.

Patients with neurogenic detrusor overactivity (NDO) and age-matched healthy controls were recruited. Concomitant medication and diseases affecting the cardio-vascular system were exclusion criteria. A 3-channel resting electrocardiogram (ECG) was recorded in supine position for 15 minutes during four consecutive visits: 1) 2 weeks prior onabotulinumtoxinA intradetrusor injections, 2) 10 minutes prior injections, 3) 30 minutes after injections, and 4) 6 weeks after injections. NDO patients received intradetrusor injections (300 units Botox®) between visits 2 and 3 . The control group had no intervention.

Short-term (5min) HRV analysis included assessment of frequency and time domain parameters. Statistical analysis was performed using ANOVA with repeated measures and the t-test. Due to multiple comparisons, a was corrected to 0.0125 (Bonferroni method).

Twelve healthy volunteers $(5$ ㅇ, $7 \hat{\delta} ; 46 \pm 12$ years old) and 12 NDO patients (5우, $7 \hat{3} ; 46 \pm 13$ years old) completed all measurements. Comparing both groups, 
resting heart rate was significantly higher in the patients group at visit 4 only. No further significant differences in time and frequency domain parameters were discovered.

Within the NDO group, standard deviation of the normal to normal intervals (SDNN) in the ECG demonstrated a significant decrease $(1.70$ to $1.53 \mathrm{~ms}$, $\mathrm{p}=0.003$ ) from visit 3 to 4 , whereas the total power (TP) significantly increased (3.05 to $3.29 \mathrm{~ms}^{2}, \mathrm{p}=0.009$ ) from visit 2 to 3 . This increase subsided until visit 4 . Study limitations: single treatment investigation under resting conditions only. In conclusion, onabotulinumtoxinA intradetrusor injections do not seem to affect resting state cardiac function. Short-term changes such as total power might rather result from natural cardio-vascular responses to the procedure itself (e.g. discomfort, stress). Further detailed investigations also under physical stress and repeated injections are necessary to fully exclude systemic cardiac side effects of onabotulinumtoxinA intradetrusor injections.

\section{KEY WORDS}

autonomic cardiac function; botulinum toxin; heart rate variability; intradetrusor injections; neurogenic detrusor overactivity; onabotulinumtoxinA; spinal cord injury; systemic side effects 


\section{INTRODUCTION}

Detrusor overactivity (DO) is a urodynamic finding that can be the underlying cause of bothersome lower urinary tract symptoms such as urinary urgency and/or incontinence. Furthermore, DO may cause irreversible alterations to morphology and function of the urinary tract, including in its worst case renal failure (Panicker, et al., 2015). First line treatment of DO is usually with antimuscarinics and just recently also a $\beta 3$ adrenergic receptor agonist has been approved for this indication (Panicker, et al., 2015).

However, antimuscarinics and/or $\beta 3$ adrenergic receptor agonists might not be sufficient to adequately reduce DO. In such cases, a second line treatment option is onabotulinumtoxinA intradetrusor injections (Panicker, et al., 2015). The efficacy of onabotulinumtoxinA intradetrusor injections has been proven previously and the safety profile seems to be beneficial (Cruz, et al., 2011, Ginsberg, et al., 2012). However, most studies reporting on safety concentrated on adverse events related to the injection procedure itself, i.e. urinary tract infection, bleeding, pain, or the local effects on the bladder, i.e. urinary retention. Distant effects after intradetrusor injections have not yet been reported or investigated systematically (Eldred-Evans, et al., 2015).

However, there is evidence that botulinum toxin causes effects related but not necessarily limited to its action on cholinergic nerve terminals elsewhere in the body than at the site of injection (Lange, et al., 1991, Roche, et al., 2008). One important neuromuscular structure that might be affected by distant effects 
following injection of botulinum toxin is the heart (Claus, et al., 1995, Girlanda, et al., 1992, Lamanna, et al., 1988, Meichsner and Reichel, 2005, Tsuboi, et al., 2002). By blocking acetylcholine release form the autonomic nerve terminals, botulinum toxin can affect 1) parasympathetic control on the sinoatrial and atrioventricular node of the heart through the vagal nerve (Dickson and Shevky, 1923) and 2) the preganglionic sympathetic innervation of the heart (Oh, et al., 2011, Tsuboi, et al., 2002). Both, sympathetic and parasympathetic influence on the heart can be assessed using heart rate variability (HRV) analysis. During normal sinus rhythm, the heart rate physiologically varies from beat to beat as a result of the dynamic interplay between the multiple physiologic mechanisms that regulate the instantaneous heart rate (Bilchick and Berger, 2006). HRV reflects the ability of the cardio-vascular system to rapidly adapt to changing needs in response to a broad range of internal and external stimuli and conditions and thus, is a measure of cardiac and overall health (Pumprla, et al., 2002). A reduced HRV has been associated with a poor prognosis of cardio-vascular disease, an increased risk of incident myocardial infarction, cardiovascular mortality, and death from other causes in the general population (Greiser, et al., 2009).

Considering that onabotulinumtoxinA is a highly potent neurotoxin and that leakage into the circulation cannot be prevented or excluded during intradetrusor injections, it was the purpose of this study to investigate the potential effects of onabotulinumtoxinA on cardiac function after intradetrusor injections using heart rate variability $(\mathrm{HRV})$ assessment. 
From the anticholinergic mechanism of onabotulinumtoxinA and previous findings (Claus, et al., 1995, Dickson and Shevky, 1923, Lamanna, et al., 1988, Meichsner and Reichel, 2005, Pokushalov, et al., 2015, Vita, et al., 1987), we would expect a reduced HRV following interdetrusor injection.

\section{SUBJECTS AND METHODS}

This is a prospective, controlled, single center study. The study was performed according to the declaration of Helsinki, approved by the local ethics committee and registered at ClinicalTrials.gov (NCT identifier: NCT01337024). All subjects provided written informed consent prior to study inclusion.

\section{Subjects}

Patients with neurogenic detrusor overactivity (NDO) eligible for treatment with onabotulinumtoxin $A$ intradetrusor injections and age-matched ( \pm 5 years) healthy controls were recruited.

Inclusion criteria for patients: age $\geq 18$ years, urodynamically proven NDO refractory to antimuscarinic treatment.

Inclusion criteria for healthy subjects: age $\geq 18$ years, good physical and mental health.

Exclusion criteria: pregnancy or lactation, any diseases of the cardiovascular system (e.g. cardiac arrhythmia, myocardial infarction, surgery), as well as cardiac pacemaker and medication with impact to cardiac function (e.g. beta- 
blockers, antiarrhythmic drugs), and medication influencing thyroid function; use of antimuscarinic therapy within 10 days prior to the first measurement; previous botulinum toxin treatment within the last 12 months prior to the first measurement.

Additional exclusion criteria for healthy subjects only: any current health problem or concomitant medication.

\section{Electrocardiogram (ECG) recordings}

The measurements were performed in a quiet separate examination room at constant room temperature and each subject / patient had a 30-35 minutes rest period to adjust to environment and setting. All subjects / patients were instructed to avoid caffeine, smoking, and meals at least $2 \mathrm{~h}$ before measurements.

Just prior to each measurement, subjects / patients were instructed to refrain from closing their eyes, moving or talking, or to intentionally control breathing.

Subsequently, a 3-channel resting ECG was recorded in a comfortable supine position with empty bladder for 15 minutes during four consecutive visits: 1) 2 weeks prior to onabotulinumtoxinA intradetrusor injections, 2) 10 minutes prior to injections, 3) 30 minutes after injections, and 4) 6 weeks after injections.

To reduce a confounding effect of pain from the onabotulinumtoxinA injection procedure on the HRV measurement, all patients rated their pain level on a visual analogue scale (VAS) before and after injection treatment. Only when the postinjection pain VAS score reached the baseline value, the post-injection ECG was recorded. 
The healthy control group had their ECG recordings at identical time intervals. The recordings of each subject / patient were performed at the same day times as the initial recording (visit 1).

\section{OnabotulinumtoxinA intradetrusor injections}

All included patients with NDO received 300 units onabotulinumtoxinA (Botox®) diluted in $30 \mathrm{~mL}$ saline and injected at 30 different sites sparing the trigone using a rigid or flexible cystoscope as described previously (Wollner and Kessler, 2011).

Local intravesical anaesthesia was applied with $60 \mathrm{~mL}$ buffered lidocaine solution (30 $\mathrm{mL}$ of $2 \%$ lidocaine and $30 \mathrm{~mL}$ of $8.4 \%$ sodiumbicarbonate) instilled $15-20$ minutes prior to onabutulinumtoxinA injection.

\section{HRV Analysis}

From the 15 minutes ECG recording, the middle 5 minutes were used for HRV analysis which was performed using SOLEASY'M (Alea Solutions, Zürich, Switzerland) as follows: a) detection of r-waves in the ECG, b) calculation of the $R R$ intervals and generation of discrete event series (DES), c) calculation of power spectra from DES d) calculation of the integral of very low frequency (VLF), low frequency (LF) and high frequency (HF) ranges. From these frequency domain parameters the total power $(\mathrm{TP}=\mathrm{VLF}+\mathrm{LF}+\mathrm{HF})$ and the $\mathrm{LF} / \mathrm{HF}$ ratio were calculated. 
For the time domain analysis, the mean heart rate at rest (rHR), the standard deviation of the normal to normal ( $\mathrm{NN}$ or RR, i.e. interval between two $\mathrm{R}$ peaks) intervals (SDNN), and the root mean square of the sum of differences between adjacent NN intervals (RMSSD) were calculated.

For more details and background on HRV previous publications elsewhere are recommended (Bilchick and Berger, 2006, Pumprla, et al., 2002, Task Force, et al., 1996).

\section{Outcome measures}

The primary outcome parameter was TP, as a general indicator for both, sympathetic and parasympathetic autonomic nervous system activity on the heart.

Secondary outcome parameters were VLF, LF, HF, LF/HF, rHR, RMSSD, and SDNN.

\section{Statistical analysis}

All data, except rHR, were log10 transformed. Statistical analysis was performed using IBM SPSS Statistics 17.0 and ANOVA with repeated measures to analyze differences within each group, i.e. between visits, and the t-test to analyze differences between both groups. Due to multiple comparisons, a was corrected to 0.0125 (Bonferroni method). All values are presented as mean \pm standard deviation (SD). 


\section{RESULTS}

Twelve patients with NDO (46 \pm 13 years old; 5 females, 7 males) and 12 healthy subjects (46 \pm 12 years old; 5 females, 7 males) were included and completed all investigations (Table 1). The cause of NDO was spinal cord injury $(n=9)$, multiple sclerosis $(n=2)$, and spina bifida $(n=1)$ (Table 1$)$.

\section{HRV parameters}

Within the control group there were no significant changes of any HRV parameter throughout the four visits.

Within the NDO patient group there was a significant increase $(p=0.009)$ in TP from visit 2 to 3 and a significant decrease $(p=0.003)$ from visit 3 to 4 (Table 2, Figure 1). The SDNN parameter decreased significantly $(p=0.003)$ from visit 3 to 4 (Table 2, Figure 2). There were no further significant changes of HRV parameters throughout the four visits.

Comparing both groups during all visits revealed a significant difference $(p=$ 0.004) in rHR at visit 4 (Table 2, Figure 3). There were no further significant differences of HRV parameters between groups (Table 2, Figures 2, 4, and 5). 


\section{DISCUSSION}

The main findings of this study are a temporary increase of TP and decrease of SDNN following onabotulinumtoxinA whereas $\mathrm{rHR}$ demonstrated a generally higher level in patients than in healthy controls. All other HRV parameters, i.e. VLF, LF, HF, LF/HF, RMSSD, did not show differences between visits, i.e. before vs after onabotulinumtoxin injections, or groups.

Considering previous findings from studies on the effect of botulinum toxin on the cardiac autonomic function (Chen, et al., 1999, Claus, et al., 1995, Meichsner and Reichel, 2005, Pokushalov, et al., 2015, Reichel, et al., 1998, Topakian, et al., 2009, Vita, et al., 1987), effects seem to be predominantly HRV depressive, i.e. decrease in coefficient of variation of heart rate (Claus, et al., 1995), R-R interval variation (Chen, et al., 1999), RMSSD (Meichsner and Reichel, 2005, Pokushalov, et al., 2015, Reichel, et al., 1998), TP (Meichsner and Reichel, 2005), and SDNN (Meichsner and Reichel, 2005, Pokushalov, et al., 2015, Vita, et al., 1987), and/or parasympatholytic (Dickson and Shevky, 1923, Rosenblum, 1966), i.e. increase in resting heart rate (Meichsner and Reichel, 2005, Pokushalov, et al., 2015, Reichel, et al., 1998, Topakian, et al., 2009) and decrease of parasympathetic related test outcomes such as 30:15 ratio (Topakian, et al., 2009, Vita, et al., 1987), E/l ratio (Meichsner and Reichel, 2005, Topakian, et al., 2009, Vita, et al., 1987), and Valsava ratio (Topakian, et al., 2009, Vita, et al., 1987). In line with that, animal studies in dogs demonstrated a significant reduction and even elimination of parasympathetic related bradycardia 
and atrial fibrillation, respectively, following botulinum toxin $A$ injections into pericardial fat pads (Oh, et al., 2011, Tsuboi, et al., 2002). An attenuation of parasympathetic modulation on the heart has also been described in studies on the autonomic cardiac effects of botulism, reporting significant declines of parasympathetic test parameters that lasted longer than in sympathetic tests (Vita, et al., 1987), elevated resting heart rate, and LF/HF ratio (Topakian, et al., 2009).

However, there is also a study reporting significant bradycardia after high dose intravenous botulinum toxin A application in different animals, i.e. mice, rats, rabbits, and dogs, with electrocardiogram alterations across all species indicating conduction defects, i.e. prolongation of the P-R, QRS, and Q-T intervals. In dogs, bilateral vagotomy and/or atropine could not prevent the bradycardia and ECG changes (Lamanna, et al., 1988). In addition, bradycardia and ECG changes were also observed in the isolated animal hearts, suggesting on the one hand that those effects are independent from respiratory related conditions and on the other hand that botulinum toxin A seems to act on local cardiac conducting structures such as artrioventricular junction, His bundle, and Purkinje fibers (Lamanna, et al., 1988).

In our study we could not observe such HRV reductive and/or parasympatholytic effects as described previously, despite investigating resting conditions only under which vagal tone prevails and variations in heart rate are largely 
dependent on vagal modulation (Task Force, et al., 1996) which would then be specifically susceptible to attenuation by botulinum toxin.

In contrary, the observed temporary increase in TP from visit 2 to 3 in the patient/onabotulinumtoxinA group would rather indicate an increase in HRV since TP constitutes from the sum of the frequency domain parameters VLF, LF, and $\mathrm{HF}$ and reflects the overall autonomic activity on the heart including sympathetic (main contributor to the LF component) as well as parasympathetic (main contributor to the HF component) cardiac modulation (Bilchick and Berger, 2006, Pumprla, et al., 2002). In this context it is noteworthy that, although not significantly, the mean values of VLF, LF, and HF increased from visit 2 to 3 in the patient group suggesting a rather uniform than a single component driven change of TP. In their sum, such insignificant changes of all frequency domain parameters might contribute to the eventually statistically significant outcome of TP. In addition, TP values were not different between patient and control group and compared to normative values from subjects of the same age group (Agelink, et al., 2001) the TP values of our patients fluctuated within a normative range. Hence, attributing this temporary change in TP to an effect of onabotulinumtoxinA appears rather unlikely.

A confounding factor that can affect HRV and might contribute to the observed transient TP changes shortly after onabotulinumtoxinA injections is the use of the lidocaine based local anesthesia prior to the injections. However, lidocaine causes very different and quite opposite changes of HRV than isolated TP 
increase (Chen, et al., 2015, Shafqat, et al., 2009), making an effect of lidocaine in this context unlikely.

The significant decrease in SDNN could be related to the onabotulinumtoxinA treatment. However, looking at the values, significance mainly results form a preceding SDNN increase until visit 3 that returns just below baseline value at visit 1 without significant difference to the baseline value.

The significant difference between groups in rHR at visit 4 results most probably from the continuously higher rHR in the patient group compared to the healthy control group which nearly became already significant at visit 2. Slight rHR decrease in the control group and slight rHR increase in the patient group at visit 4 were sufficient to cause the statistical significance whereas each group demonstrated a quite stable rHR throughout all visits. Since most patients suffered from spinal cord injury, the higher rHR in the patient group might be a consequence of the altered autonomic nervous system function in such patients (Garstang and Miller-Smith, 2007).

In summary, we did not find relevant HRV changes related to onabotulinumtoxinA intradetrusor injections neither within the treated patient group nor in comparison with the untreated age-matched healthy control group. To the best of our knowledge, this is the first study on the relationship of onabotulinumtoxinA intradetrusor injections and autonomic cardiac control. There are some studies 
that investigated the effect of botulinum toxin A on cardiac autonomic function using HRV before and after injections for the treatment of cervical dystonia (Claus, et al., 1995, Girlanda, et al., 1992, Meichsner and Reichel, 2005, Nebe, et al., 1996, Tiple, et al., 2008), spastic hemiplegia (Invernizzi, et al., 2015), and other dystonic conditions (Girlanda, et al., 1992, Meichsner and Reichel, 2005). However, the results are conflicting on weather botulinum toxin A injections have an effect on autonomic cardiovascular function (Claus, et al., 1995, Girlanda, et al., 1992, Meichsner and Reichel, 2005) or not (Invernizzi, et al., 2015, Nebe, et al., 1996, Tiple, et al., 2008). Comparison amongst studies remains very difficult and needs to be done with caution due to the different injection doses, injection sites, botulinum toxin A formulations, and primary HRV endpoints used. Moreover, time point of $\mathrm{HRV}$ assessment in relation to botulinum toxin $\mathrm{A}$ injections as well as the course of treatment, i.e. single primary treatment vs. chronic repeated treatment might be additional and essential factors influencing study outcome. Post treatment measurements followed mainly within 10 days to 6 weeks after botulinum toxin A injections. In our study we re-assessed the patients 6 weeks after onabotulinumtoxinA injections since this is the usual interval within which onabotulinumtoxinA is expected to show full efficacy on the detrusor. However, an earlier occurrence of transient effects might be missed whereas 30 min after onabotulinumtoxinA (visit 3) might be too early to observe any onabotulinumtoxinA related effects (Garner, et al., 1993, Girlanda, et al., 1992). Regarding the treatment course, our patients had previous onabotulinumtoxinA treatments but not within 12 months prior to the first HRV 
measurement. Nevertheless, we cannot fully exclude a sustained onabotulinumtoxinA effect on autonomic cardiac control from previous injections. In this context it is noteworthy that in two of three studies, that demonstrated effects of botulinum toxin A on HRV, only treatment naive patients were included (in the third study, patient status was not indicated) (Claus, et al., 1995, Girlanda, et al., 1992) although short-term (14-45 days) re-injections were necessary to record significant effects. This might suggest on the one hand a dose dependent effect of botulinum toxin A on cardiac autonomic control and on the other hand that patients with repeat botulinum toxin A injections might no longer show acute effects on HRV potentially due to an already altered baseline. In contrary, Nebe et al. investigated treatment naive patients only as well but did not find any botulinum toxin A effect on HRV (Nebe, et al., 1996). Moreover, in our patient group, potential chronic adaptations of the cardiac autonomic control due to previously repeated onabotulinumtoxinA injections must be subtle enough not to result in any difference compared to the healthy control group. Despite using a quite high dosage of 300 units onabotulinumtoxinA compared to the studies indicating botulinum toxin A effects on HRV (20-130 units onabotulinumtoxinA or 500 units abobotulinumtoxinA), we could not confirm effects of onabotulinumtoxinA on HRV which is in line with the most recent study on this topic using 600 units of incobotulinumtoxinA for treatment of spastic hemiplegia in stroke survivors without significant posttreatment effects on HRV (Invernizzi, et al., 2015). A possible explanation could be a less extensive spread of onabotulinumtoxinA after intradetrusor injections compared to injections into 
striated muscle as suggested by a pilot study of Schnitzler et al. using single fiber electromyography to assess the neuromuscular jitter as sign of distant neuromuscular effects in 21 patients after 300 units onabotulinumtoxinA intradetrusor injections for NDO (Schnitzler, et al., 2011).

In view of the rather sparse body of literature in contrast to the many unclarified aspects on distant effects of botulinum toxin A after injection treatments further investigations on this topic are indicated to improve the knowledge and patient safety regarding side effects of one of the most potent neurotoxins, that is frequently used in neurorehabilitation.

Limitations of the study are: A) Focus on resting state HRV investigations only. Assessment of cardiovascular parameters under functional tasks such as tilt or exercise might have revealed different results. B) Investigation of single treatment only. No conclusions possible on the effect of repeated injections which might have altered the outcome of HRV measurements, specifically if performed at short intervals, i.e. $\leq 3$ months. C) Post-treatment assessment of onabotulinumtoxinA effects on HRV at only two time points.

In conclusion, this is the first study assessing the effects of onabotulinumtoxinA intradetrusor injections on autonomic cardiac function including 4 visits (two before and two after treatment) to control for natural fluctuations in HRV and using a healthy control group. 
Our findings indicate that onabotulinumtoxinA intradetrusor injections (300 units Botox) do not affect the resting autonomic nervous system control of cardiac function. This is highly relevant in regard to treatment of DO in patients with altered autonomic cardiac control and might influence the choice of treatment in regard to alternative treatments with systemic side effects on the heart (Schiffers, et al., 2010). Studies including HRV measurements under physical stress and after repeated onabotulinumtoxinA intradetrusor injections are desirable future tasks.

\section{ACKNOWLEDGMENTS}

This study was funded by the Swiss National Science Foundation (\#127477).

\section{REFERENCES:}

1. Agelink, M. W., Malessa, R., Baumann, B., Majewski, T., Akila, F., Zeit, T., and Ziegler, D., 2001. Standardized tests of heart rate variability: normal ranges obtained from 309 healthy humans, and effects of age, gender, and heart rate. Clin Auton Res 11, 99-108.

2. Bilchick, K. C., and Berger, R. D., 2006. Heart rate variability. J Cardiovasc Electrophysiol 17, 691-694.

3. Chen, J. T., Chen, C. C., Lin, K. P., Wang, S. J., Wu, Z. A., and Liao, K. K., 1999. Botulism: heart rate variation, sympathetic skin responses, and plasma norepinephrine. The Canadian journal of neurological sciences. Le journal canadien des sciences neurologiques 26, 123-126.

4. Chen, Y. Q., Jin, X. J., Liu, Z. F., and Zhu, M. F., 2015. Effects of stellate ganglion block on cardiovascular reaction and heart rate variability in elderly 
patients during anesthesia induction and endotracheal intubation. Journal of clinical anesthesia 27, 140-145.

5. Claus, D., Druschky, A., and Erbguth, F., 1995. Botulinum toxin: influence on respiratory heart rate variation. Movement disorders : official journal of the Movement Disorder Society 10, 574-579.

6. Cruz, F., Herschorn, S., Aliotta, P., Brin, M., Thompson, C., Lam, W., Daniell, G., Heesakkers, J., and Haag-Molkenteller, C., 2011. Efficacy and safety of onabotulinumtoxinA in patients with urinary incontinence due to neurogenic detrusor overactivity: a randomised, double-blind, placebo-controlled trial. European urology 60, 742-750.

7. Dickson, E. C., and Shevky, R., 1923. Botulism. Studies on the Manner in Which the Toxin of Clostridium Botulinum Acts Upon the Body : I. The Effect Upon the Autonomic Nervous System. The Journal of experimental medicine 37, 711-731.

8. Eldred-Evans, D., Seth, J., Khan, M. S., Chapple, C. R., Dasgupta, P., and Sahai, A., Adverse Events with Botox and Dysport for refractory overactive bladder: A systematic review 45th Annual Meeting of the International Continence Society (ICS), 6-9 October 2015, , Montreal, Canada, 2015, pp. 105 - 106.

9. Garner, C. G., Straube, A., Witt, T. N., Gasser, T., and Oertel, W. H., 1993. Time course of distant effects of local injections of botulinum toxin. Movement disorders : official journal of the Movement Disorder Society 8, 33-37.

10. Garstang, S. V., and Miller-Smith, S. A., 2007. Autonomic nervous system dysfunction after spinal cord injury. Phys Med Rehabil Clin N Am 18, 275-296, vi-vii.

11. Ginsberg, D., Gousse, A., Keppenne, V., Sievert, K. D., Thompson, C., Lam, W., Brin, M. F., Jenkins, B., and Haag-Molkenteller, C., 2012. Phase 3 efficacy and tolerability study of onabotulinumtoxinA for urinary incontinence from neurogenic detrusor overactivity. The Journal of urology 187, 2131-2139.

12. Girlanda, P., Vita, G., Nicolosi, C., Milone, S., and Messina, C., 1992. Botulinum toxin therapy: distant effects on neuromuscular transmission and autonomic nervous system. Journal of neurology, neurosurgery, and psychiatry 55, 844-845.

13. Greiser, K. H., Kluttig, A., Schumann, B., Swenne, C. A., Kors, J. A., Kuss, O., Haerting, J., Schmidt, H., Thiery, J., and Werdan, K., 2009. Cardiovascular diseases, risk factors and short-term heart rate variability in an elderly general population: the CARLA study 2002-2006. Eur J Epidemiol 24, 123-142.

14. Invernizzi, M., Carda, S., Molinari, C., Stagno, D., Cisari, C., and Baricich, A., 2015. Heart Rate Variability (HRV) modifications in adult hemiplegic patients after botulinum toxin type A (nt-201) injection. European journal of physical and rehabilitation medicine 51, 353-359.

15. Lamanna, C., el-Hage, A. N., and Vick, J. A., 1988. Cardiac effects of botulinal toxin. Archives internationales de pharmacodynamie et de therapie 293, 69-83.

16. Lange, D. J., Rubin, M., Greene, P. E., Kang, U. J., Moskowitz, C. B., Brin, M. F., Lovelace, R. E., and Fahn, S., 1991. Distant effects of locally injected 
botulinum toxin: a double-blind study of single fiber EMG changes. Muscle \& nerve $14,672-675$.

17. Meichsner, M., and Reichel, G., 2005. [Effect of botulinum toxin a and B on vegetative cardiac innervation]. Fortschritte der Neurologie-Psychiatrie 73, 409414.

18. Nebe, A., Schelosky, L., Wissel, J., Ebersbach, G., Scholz, U., and Poewe, W., 1996. No effects on heart-rate variability and cardiovascular reflex tests after botulinum toxin treatment of cervical dystonia. Movement disorders : official journal of the Movement Disorder Society 11, 337-339.

19. Oh, S., Choi, E. K., Zhang, Y., and Mazgalev, T. N., 2011. Botulinum toxin injection in epicardial autonomic ganglia temporarily suppresses vagally mediated atrial fibrillation. Circulation. Arrhythmia and electrophysiology 4, 560-565.

20. Panicker, J. N., Fowler, C. J., and Kessler, T. M., 2015. Lower urinary tract dysfunction in the neurological patient: clinical assessment and management. The Lancet. Neurology 14, 720-732.

21. Pokushalov, E., Kozlov, B., Romanov, A., Strelnikov, A., Bayramova, S., Sergeevichev, D., Bogachev-Prokophiev, A., Zheleznev, S., Shipulin, V., Lomivorotov, V. V., Karaskov, A., Po, S. S., and Steinberg, J. S., 2015. LongTerm Suppression of Atrial Fibrillation by Botulinum Toxin Injection Into Epicardial Fat Pads in Patients Undergoing Cardiac Surgery: One-Year FollowUp of a Randomized Pilot Study. Circulation. Arrhythmia and electrophysiology 8, 1334-1341.

22. Pumprla, J., Howorka, K., Groves, D., Chester, M., and Nolan, J., 2002. Functional assessment of heart rate variability: physiological basis and practical applications. Int J Cardiol 84, 1-14.

23. Reichel, G., Stenner, A., and Doberenz, M., 1998. Therapie mit Botulinum-Toxin A. Besteht die Gefahr vegetativer kardialer Störungen? Jatros Neuro 14, 49 - 52.

24. Roche, N., Schnitzler, A., Genet, F. F., Durand, M. C., and Bensmail, D., 2008. Undesirable distant effects following botulinum toxin type a injection. Clinical neuropharmacology 31, 272-280.

25. Rosenblum, I., 1966. Effects of Cl. botulinum type A toxin on the isolated cat heart. Archives internationales de pharmacodynamie et de therapie 159, 281-287.

26. Schiffers, M., Sauermann, P., Schurch, B., and Mehnert, U., 2010. The effect of tolterodine 4 and $8 \mathrm{mg}$ on the heart rate variability in healthy subjects. World journal of urology 28, 651-656.

27. Schnitzler, A., Genet, F., Durand, M. C., Roche, N., Bensmail, D., ChartierKastler, E., and Denys, P., 2011. Pilot study evaluating the safety of intradetrusor injections of botulinum toxin type A: investigation of generalized spread using single-fiber EMG. Neurourology and urodynamics 30, 1533-1537.

28. Shafqat, K., Pal, S. K., Kumari, S., and Kyriacou, P. A., 2009. Time-frequency analysis of HRV data from locally anesthetized patients. Conference proceedings : ... Annual International Conference of the IEEE Engineering in Medicine and 
Biology Society. IEEE Engineering in Medicine and Biology Society. Annual Conference 2009, 1824-1827.

29. Task Force, of The European Society of Cardiology, and The North American Society of Pacing and Electrophysiology, 1996. Heart rate variability. Standards of measurement, physiological interpretation, and clinical use. Task Force of the European Society of Cardiology and the North American Society of Pacing and Electrophysiology. European heart journal 17, 354-381.

30. Tiple, D., Strano, S., Colosimo, C., Fabbrini, G., Calcagnini, G., Prencipe, M., and Berardelli, A., 2008. Autonomic cardiovascular function and baroreflex sensitivity in patients with cervical dystonia receiving treatment with botulinum toxin type A. Journal of neurology 255, 843-847.

31. Topakian, R., Heibl, C., Stieglbauer, K., Dreer, B., Nagl, M., Knoflach, P., and Aichner, F. T., 2009. Quantitative autonomic testing in the management of botulism. Journal of neurology 256, 803-809.

32. Tsuboi, M., Furukawa, Y., Kurogouchi, F., Nakajima, K., Hirose, M., and Chiba, S., 2002. Botulinum neurotoxin A blocks cholinergic ganglionic neurotransmission in the dog heart. Japanese journal of pharmacology 89, 249254.

33. Vita, G., Girlanda, P., Puglisi, R. M., Marabello, L., and Messina, C., 1987. Cardiovascular-reflex testing and single-fiber electromyography in botulism. A longitudinal study. Archives of neurology 44, 202-206.

34. Wollner, J., and Kessler, T. M., 2011. Botulinum toxin injections into the detrusor. BJU international 108, 1528-1537.

\section{FIGURE LEGENDS}

Figure 1: Total power (TP, in $\left.\mathrm{ms}^{2}\right)$ during the four consecutive visits (1-4) for the control (blue bars) and the neurogenic detrusor overactivity (NDO, grey bars) group. In the NDO group, there was a significant $\left({ }^{*} p=0.009\right)$ increase in TP from visit 2 to 3 and significant $\left({ }^{\#} p=0.003\right)$ decrease from visit 3 to 4 .

Figure 2: Root mean square of the successive differences (RMSSD, in ms) and standard deviation of $\mathrm{NN}$ intervals (SDNN, in ms) during the four consecutive visits (1-4) for the control (blue bars) and the neurogenic detrusor overactivity 
(NDO, grey bars) group. In the NDO group, SDNN decreased significantly ( ${ }^{\#} \mathrm{p}=$ 0.003) from visit 3 to 4.

Figure 3: Resting heart rate ( $\mathrm{rHT}$, in bpm) during the four consecutive visits (1-4) for the control (blue bars) and the neurogenic detrusor overactivity (NDO, grey bars) group. Comparing the control versus the NDO group, there was a significant difference $\left({ }^{\#} \mathrm{p}=0.004\right)$ in rHR at visit 4 .

Figure 4: Low frequency spectrum $\left(\mathrm{LF}\right.$, in $\left.\mathrm{ms}^{2}\right)$ and high frequency spectrum (HF, in $\mathrm{ms}^{2}$ ) during the four consecutive visits (1-4) for the control (blue bars) and the neurogenic detrusor overactivity (NDO, grey bars) group.

Figure 5: Low frequency / high frequency (LF/HF) ratio and very low frequency spectrum (VLF, in $\mathrm{ms}^{2}$ ) during the four consecutive visits (1-4) for the control (blue bars) and the neurogenic detrusor overactivity (NDO, grey bars) group. 
Table 1: Demographic data of neurogenic detrusor overactivity (NDO) patients and subjects.

\begin{tabular}{|c|c|c|c|c|}
\hline $\begin{array}{l}\text { NDO } \\
\text { patients }\end{array}$ & Neurological lesion/disease & $\begin{array}{c}\text { Duration of } \\
\text { lesion/disease [years] }\end{array}$ & Age [years] & Gender \\
\hline 1 & Spinal cord inury, TH5, AIS A & 18 & 36 & $\mathrm{M}$ \\
\hline 2 & Spinal cord inury, TH6, AIS A & 17 & 39 & $\mathrm{M}$ \\
\hline 3 & Multiple sclerosis & 21 & 43 & $\mathrm{~F}$ \\
\hline 4 & Spinal cord inury, TH5, AIS A & 4 & 43 & $\mathrm{M}$ \\
\hline 5 & Spinal cord inury, TH6, AIS A & 8 & 38 & $M$ \\
\hline 6 & Spinal cord inury, TH3, AIS A & 24 & 71 & $\mathrm{~F}$ \\
\hline 7 & Spinal cord inury, TH4, AIS C & 7 & 51 & $M$ \\
\hline 8 & Spinal cord inury, L4, AIS C & 3 & 47 & $\mathrm{~F}$ \\
\hline 9 & Spinal cord inury, C1, AIS D & 3 & 63 & $M$ \\
\hline 10 & Multiple sclerosis & 33 & 58 & $\mathrm{~F}$ \\
\hline 11 & Spinal cord inury, L2, AIS A & 27 & 38 & $\mathrm{~F}$ \\
\hline 12 & Meningomyelocele & 22 & 22 & $M$ \\
\hline \multicolumn{5}{|l|}{$\begin{array}{l}\text { Healthy } \\
\text { controls }\end{array}$} \\
\hline 1 & None & - & 35 & $M$ \\
\hline 2 & None & - & 34 & $M$ \\
\hline 3 & None & - & 48 & $\mathrm{~F}$ \\
\hline 4 & None & - & 48 & $M$ \\
\hline 5 & None & - & 41 & $\mathrm{~F}$ \\
\hline 6 & None & - & 67 & $M$ \\
\hline 7 & None & - & 51 & $\mathrm{M}$ \\
\hline 8 & None & - & 50 & $\mathrm{~F}$ \\
\hline 9 & None & - & 62 & $M$ \\
\hline 10 & None & - & 54 & $\mathrm{M}$ \\
\hline 11 & None & - & 36 & $\mathrm{~F}$ \\
\hline 12 & None & - & 27 & $\mathrm{~F}$ \\
\hline
\end{tabular}

AIS American spinal injury association Impairment Scale; NDO neurogenic detrusor overactivity 
Table 2: Heart rate variability (HRV) outcome parameters (mean \pm SD) from all 4 consecutive visits of both groups, patients undergoing onabotulinumtoxinA (onaBTA) intradetrusor injections for treatment of neurogenic detrusor overactivity $(n=12)$ and age-matched healthy controls (HC, $n=12)$. All HRV parameters were generated from a 5 minutes time intervals and, except resting heart rate (rHR), log10 transformed. The p-values result form a t-test analysis between both groups. Due to multiple comparison a was corrected to 0.0125 for all values (Bonferroni method).

\begin{tabular}{|c|c|c|c|c|c|c|c|c|c|c|c|c|}
\hline & \multicolumn{3}{|c|}{ Visit 1} & \multicolumn{3}{|c|}{ Visit 2} & \multicolumn{3}{|c|}{ Visit 3} & \multicolumn{3}{|c|}{ Visit 4} \\
\hline & onaBTA & $\mathrm{HC}$ & $p$ value & onaBTA & $\mathrm{HC}$ & $p$ value & onaBTA & $\mathrm{HC}$ & $p$ value & onaBTA & $\mathrm{HC}$ & $p$ value \\
\hline rHR [bpm] & $71 \pm 15$ & $65 \pm 10$ & 0.203 & $73 \pm 17$ & $62 \pm 9$ & 0.062 & $69 \pm 14$ & $60 \pm 8$ & 0.074 & $73 \pm 10$ & $61 \pm 9$ & 0.004 \\
\hline $\operatorname{VLF}\left[\mathrm{ms}^{2}\right]$ & $2.46 \pm 0.4$ & $2.55 \pm 0.4$ & 0.608 & $2.66 \pm 0.3$ & $2.56 \pm 0.3$ & 0.489 & $2.90 \pm 0.4$ & $2.61 \pm 0.3$ & 0.053 & $2.63 \pm 0.4$ & $2.80 \pm 0.4$ & 0.312 \\
\hline $\mathrm{LF} \quad\left[\mathrm{ms}^{2}\right]$ & $2.50 \pm 0.4$ & $2.37 \pm 0.4$ & 0.413 & $2.51 \pm 0.5$ & $2.54 \pm 0.4$ & 0.857 & $2.77 \pm 0.4$ & $2.49 \pm 0.4$ & 0.131 & $2.42 \pm 0.5$ & $2.55 \pm 0.5$ & 0.575 \\
\hline $\mathrm{HF}\left[\mathrm{ms}^{2}\right]$ & $2.29 \pm 0.6$ & $2.32 \pm 0.4$ & 0.876 & $2.27 \pm 0.6$ & $2.33 \pm 0.5$ & 0.808 & $2.51 \pm 0.6$ & $2.42 \pm 0.5$ & 0.674 & $2.12 \pm 0.7$ & $2.46 \pm 0.5$ & 0.181 \\
\hline LF/HF & $1.13 \pm 0.2$ & $1.03 \pm 0.1$ & 0.160 & $1.15 \pm 0.2$ & $1.12 \pm 0.2$ & 0.790 & $1.13 \pm 0.2$ & $1.04 \pm 0.2$ & 0.247 & $1.18 \pm 0.2$ & $1.05 \pm 0.2$ & 0.073 \\
\hline $\mathrm{TP}\left[\mathrm{ms}^{2}\right]$ & $2.96 \pm 0.4$ & $2.95 \pm 0.4$ & 0.974 & $3.05 \pm 0.4$ & $3.00 \pm 0.3$ & 0.769 & $3.29 \pm 0.3$ & $3.05 \pm 0.3$ & 0.046 & $3.04 \pm 0.4$ & $3.22 \pm 0.4$ & 0.257 \\
\hline RMSSD [ms] & $1.45 \pm 0.4$ & $1.39 \pm 0.3$ & 0.707 & $1.49 \pm 0.4$ & $1.41 \pm 0.2$ & 0.552 & $1.57 \pm 0.3$ & $1.53 \pm 0.3$ & 0.795 & $1.32 \pm 0.3$ & $1.51 \pm 0.3$ & 0.134 \\
\hline SDNN [ms] & $1.59 \pm 0.3$ & $1.53 \pm 0.2$ & 0.584 & $1.65 \pm 0.2$ & $1.57 \pm 0.2$ & 0.316 & $1.70 \pm 0.1$ & $1.64 \pm 0.2$ & 0.454 & $1.53 \pm 0.2$ & $1.62 \pm 0.2$ & 0.278 \\
\hline
\end{tabular}

HC healthy controls, HF high frequency, LF low frequency, LF/HF low frequency / high frequency ratio, onaBTA onabotulinumtoxinA, rHR resting heart rate, RMSSD Root Mean Square of the Successive Differences, SDNN standard deviation of the NN intervals, TP total power, VLF very low frequency. 


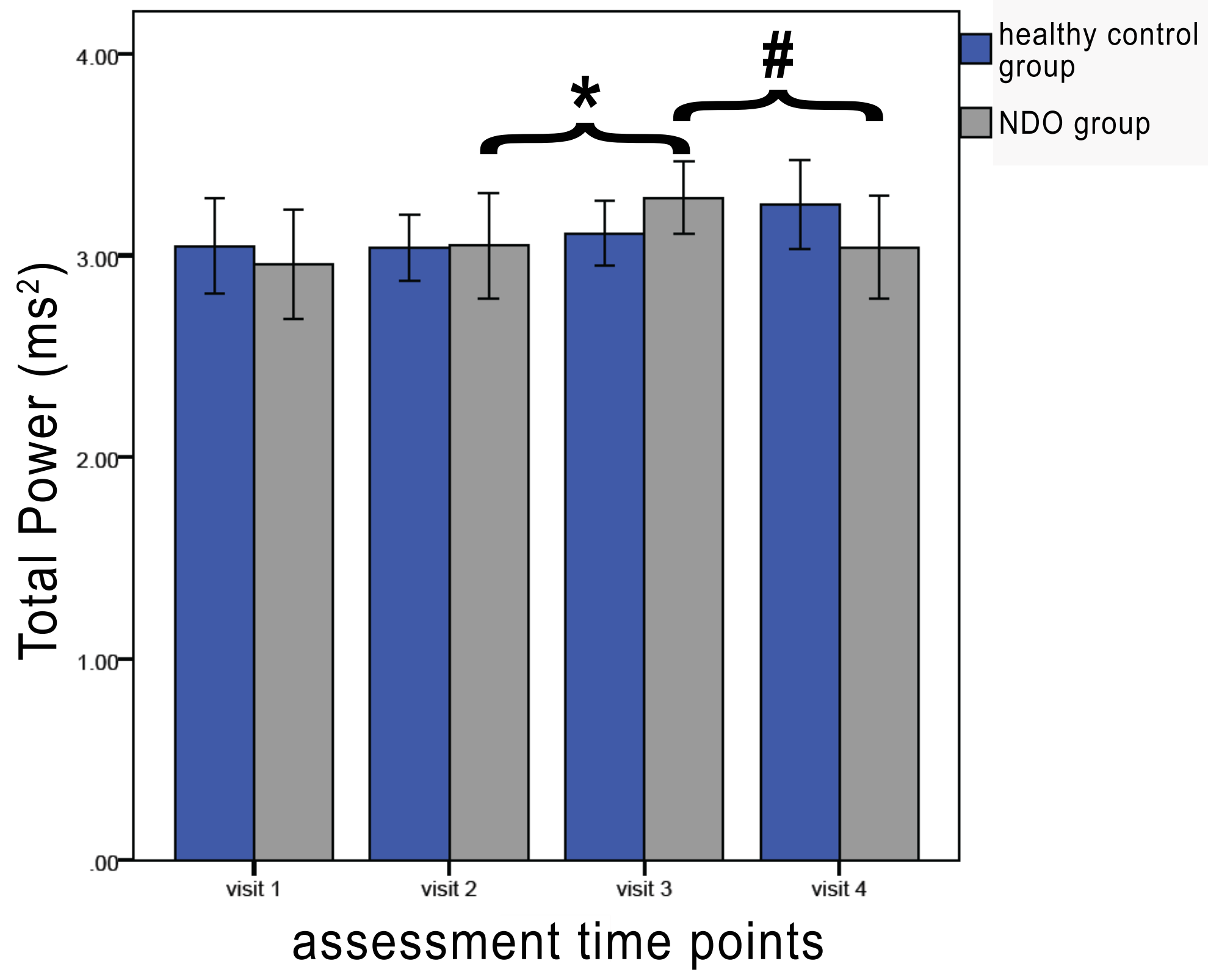




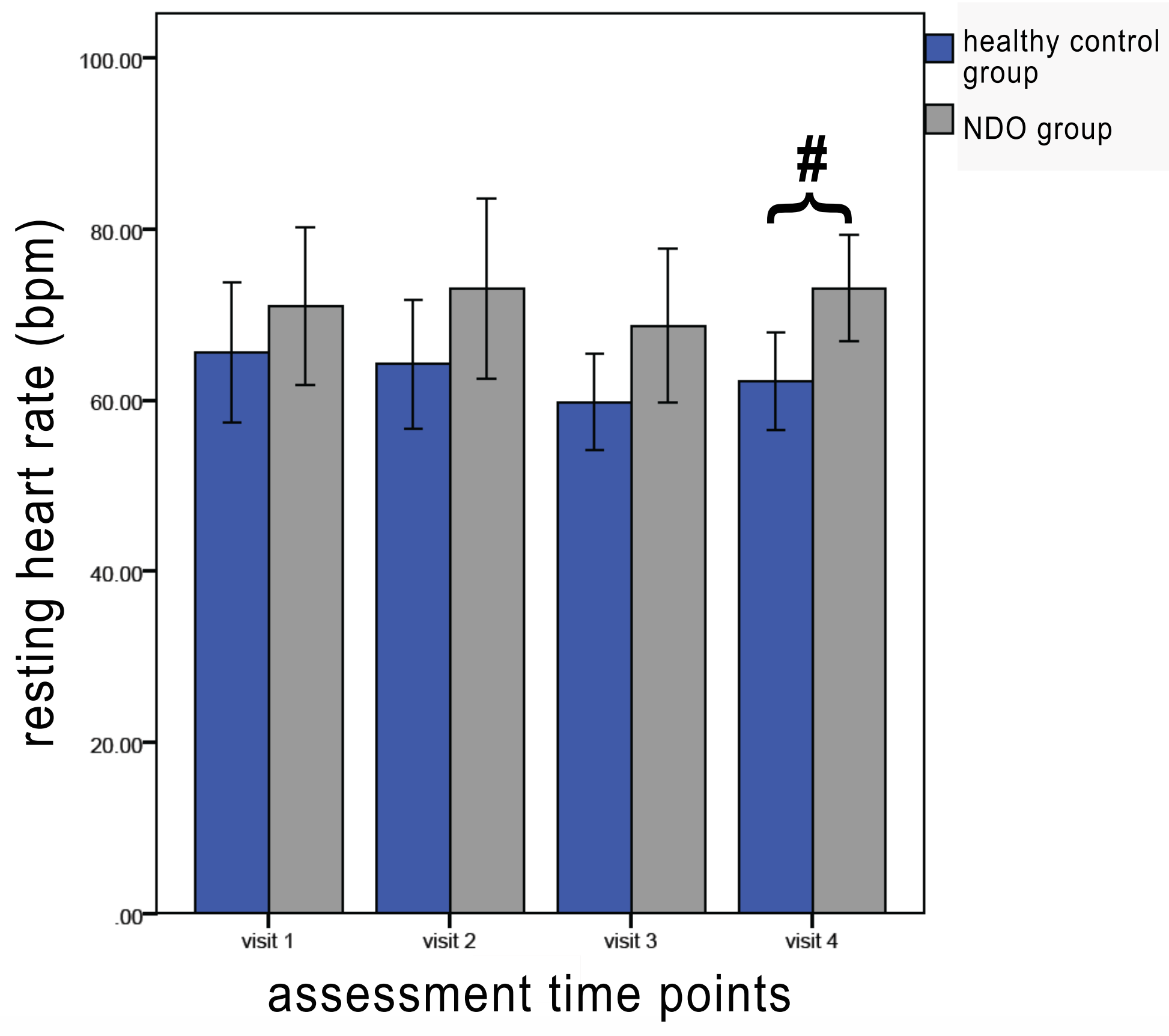




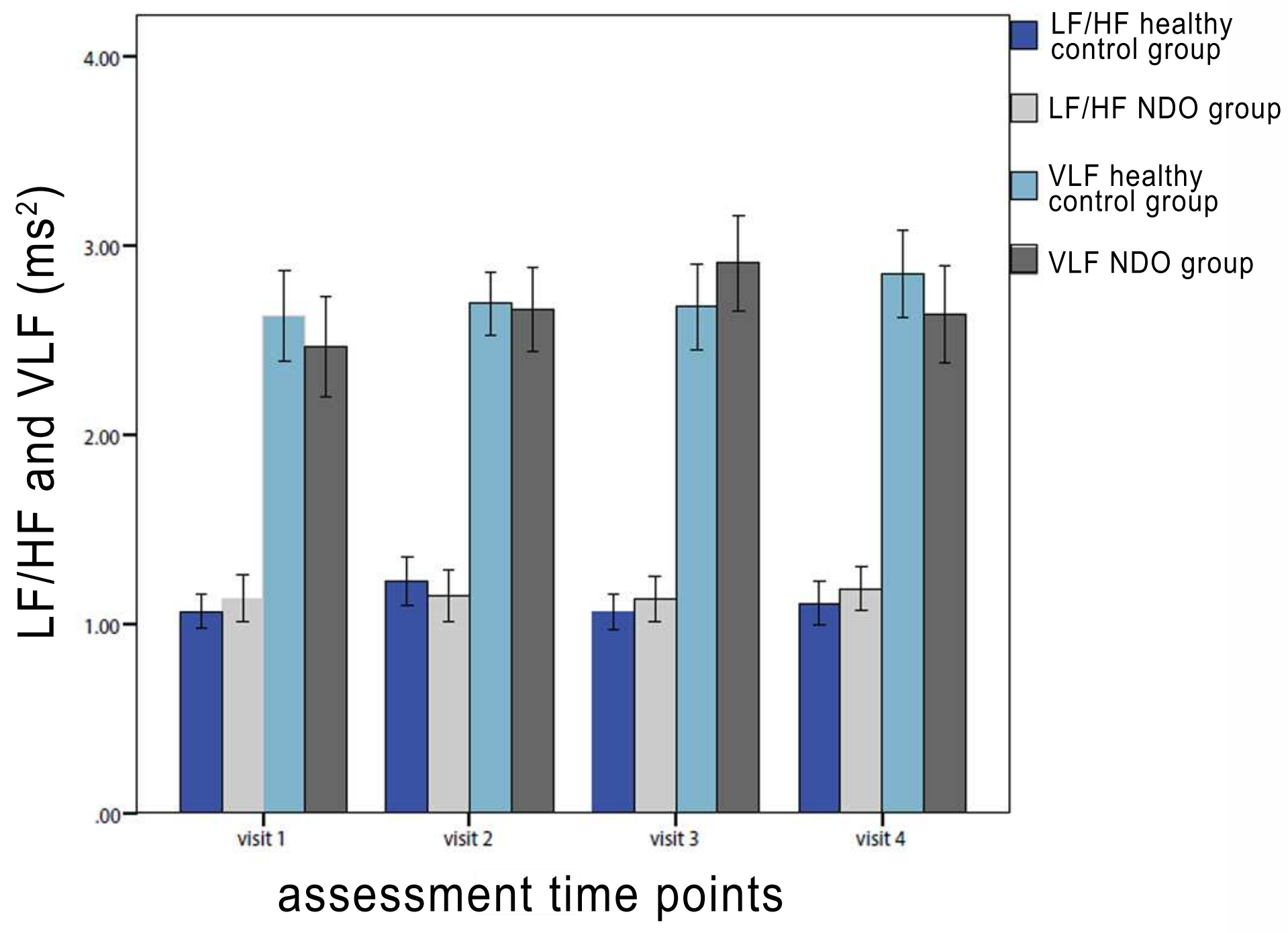

